\title{
Polymorphisms in the Osteopontin Are Associated with Susceptibility to Ankylosing Spondylitis in a Han Chinese Population
}

\author{
Juyi Li, ${ }^{1}$ Yi Cai, ${ }^{2}$ Zhongjing Wang, ${ }^{3}$ Aiping Deng $\mathbb{D}^{1},{ }^{1}$ and Guoliang Yang ${ }^{4}{ }^{4}$ \\ ${ }^{1}$ Department of Pharmacy, The Central Hospital of Wuhan, Tongji Medical College, Huazhong University of Science and Technology, \\ Wuhan, Hubei, China \\ ${ }^{2}$ Department of Pain, The Central Hospital of Wuhan, Tongji Medical College, Huazhong University of Science and Technology, \\ Wuhan, Hubei, China \\ ${ }^{3}$ Department of Endocrinology, The Central Hospital of Wuhan, Tongji Medical College, \\ Huazhong University of Science and Technology, Wuhan, Hubei, China \\ ${ }^{4}$ Department of Information, The Central Hospital of Wuhan, Tongji Medical College, Huazhong University of Science and Technology, \\ Wuhan, Hubei, China
}

Correspondence should be addressed to Aiping Deng; dapyxb@163.com and Guoliang Yang; 1625657794@qq.com

Received 26 October 2017; Accepted 20 December 2017; Published 18 January 2018

Academic Editor: Ewa Mojs

Copyright (C) 2018 Juyi Li et al. This is an open access article distributed under the Creative Commons Attribution License, which permits unrestricted use, distribution, and reproduction in any medium, provided the original work is properly cited.

\begin{abstract}
The aim of this study was to investigate whether osteopontin (OPN) variants are associated with susceptibility to ankylosing spondylitis (AS) in a Chinese population. Polymorphisms at the 9175th position in exon 7 of OPN and rs17524488 were genotyped using direct sequencing in 186 unrelated AS patients and 188 ethnically matched healthy controls. Serum concentration of OPN was measured by enzyme-linked immunosorbent assay (ELISA) in all participants. AS patients displayed significantly higher OPN serum levels than the controls $(P<001)$. A heterozygous, novel $9175 \mathrm{~T}>\mathrm{A}$ in exon 7 of the $O P N$ gene was found in this study. In healthy controls, subjects carrying the rs $17524488 \mathrm{G} / \mathrm{G}$ genotype of the OPN display significantly higher OPN serum levels than the GG/GG genotype $(P<0.05)$. Plasma OPN level is implicated as an early diagnostic marker of AS. The novel 9175th- (exon 7) position polymorphism of $O P N$ and rs17524488 were related to susceptibility to AS in a Chinese population, the rs17524488 G/G genotype may be involved in the pathogenesis of AS, and the precise molecular mechanism underlying the influence of OPN polymorphisms on the development of AS remains to be determined in the further prospective studies.
\end{abstract}

\section{Introduction}

Ankylosing spondylitis (AS) is an autoimmune disease caused by chronic inflammation response and pathological mineralization and usually strikes the males $[1,2]$. Incipient symptoms of AS appear before 40 years of and it is characterized by inflammatory back pain and the stiffness and ankylosis of spinal joints [3]. Approximately $0.2-1.4 \%$ of the general population suffers from AS, and its incidence is $0.2-0.54 \%$ in the Chinese $[2,4]$.

AS is highly heritable and many genetic polymorphisms have been reported to correlate the onset of AS [5]. HLA B27 has been reported to be as a major genetic contributor to AS and $90 \%$ AS patients are accompanied with the positive HLA B27 [6]; in addition, there are other genetic polymorphisms, such as JMY, PTGER4, JARIDIA, and ANTXR2 [7, 8]. Although many studies focus on the pathogenesis of AS, the etiology and mechanisms behind AS remain unclear.

Osteopontin (OPN) is a secreted phosphoglycoprotein with several functions in different physiological and pathological processes, including bone remodeling process, inflammation, autoimmune responses, and tumorigenesis [9]. Studies have shown that OPN serum levels are higher in some kinds of autoimmune diseases than healthy controls and may influence development of these diseases through enhancing the proinflammatory $\mathrm{T}$ helper type 1 (TH1) and $\mathrm{TH} 17$ cell 
responses and inhibiting the $\mathrm{TH} 2$ responses [10]. More than 10 SNPs have been identified in the OPN promoter. These polymorphisms may affect the transcriptional activity of $O P N$ and some of them are thought to be genetic risk factors for disease susceptibility [11-13], of which the rs17524488 (-156 $\mathrm{GG} / \mathrm{G}$ ) polymorphism is most frequently studied, which has been found to associate with several diseases, including hip osteoarthritis [14], cancer [15], and diabetic nephropathy [16].

These observations led us to hypothesize that OPN polymorphisms may be involved in the pathogenesis of AS through inflammation and/or the bone remodeling process. However, there have been little studies investigating the association of the OPN polymorphisms and AS to date. Therefore, the aim of this study was to investigate if an association exists between OPN polymorphisms, OPN serum levels, and the risk of AS in a Chinese population.

\section{Materials and Methods}

2.1. Subjects. From May 2010 to October 2013, 188 healthy volunteers for physical examination and 186 unrelated AS patients were recruited from the Affiliated Hospital of Ningxia Medical University in a Han Chinese population. All AS patients are HLA-B27 positive. Patients diagnosed with AS according to the modified New York criteria developed in 1984 were included in the study. All of the subjects gave written informed consent. This study was approved by the Ethics Committee of Ningxia Medical University. Two mL fasting venous blood was collected from all subjects; one $\mathrm{mL}$ full blood sample was used to extract genomic DNA; however, the other one $\mathrm{mL}$ full blood sample was centrifuged (10 minutes at $1160 \mathrm{~g}$ ) to separate the clot material from the solution phase (serum) which was stored at $-80^{\circ} \mathrm{C}$ for further analysis. Genomic DNA was extracted using TIANGEN reagent set (Beijing) following standard protocols; DNA samples were stored at $-20^{\circ} \mathrm{C}$. Exclusion criteria were patients with diabetes mellitus, cancer, severe liver and kidney failure, and being on therapy for any chronic inflammatory disease. Healthy volunteers as the control group were recruited from the department of physical examination at the same time, and those carrying HLA-B (rs13202464) were excluded.

2.2. Information Collection. The following information was collected: sex, age, duration of disease, body mass index (BMI), smoking status, family history of AS, and HLAB27. The Bath Ankylosing Spondylitis Disease Activity Index (BASDAI), Bath Ankylosing Spondylitis Functional Index (BASFI), and Bath Ankylosing Spondylitis Global (BAS-G) score were applied to evaluate the disease activity, physical function, and global wellbeing, respectively. The modified Chinese versions of BASDAI, BASFI, and BAS-G have good intraclass correlation and Cronbach's alpha [17].

2.3. Analysis of Polymorphisms in the OPN Regulatory Region. The 9175th position in exon 7 of $O P N$ and rs17524488 variants were genotyped by direct sequencing of the sense and antisense strands following polymerase chain reaction (PCR) amplification. A primer pair of the 9175th position was 5' -TACCCTGATGCTACAGACGAGG-3 (forward) and
TABLE 1: Characteristics of AS patients and healthy control (mean \pm SEM).

\begin{tabular}{lccc}
\hline Variable & Case (\%) & Control (\%) & $P$ \\
\hline Gender & & & \\
$\quad$ Male & $96(51.61)$ & $100(53.19)$ & 0.760 \\
$\quad$ Female & $90(48.39)$ & $88(46.81)$ & \\
Age (years) & $27.24 \pm 0.68$ & $28.90 \pm 0.68$ & 0.084 \\
BMI $\left(\mathrm{kg} / \mathrm{m}^{2}\right)$ & $24.68 \pm 0.29$ & $25.17 \pm 0.21$ & 0.166 \\
Disease duration (years) & $5.67 \pm 0.28$ & - & - \\
BASDAI (0-10) & $4.23 \pm 0.06$ & - & - \\
BASFI $(0-10)$ & $2.79 \pm 0.06$ & - & - \\
BAS-G (0-10) & $4.38 \pm 0.09$ & - & - \\
Family history & & & \\
$\quad$ Yes & $25(13.44)$ & $12(6.38)$ & 0.022 \\
$\quad$ No & $161(86.56)$ & $176(93.62)$ & \\
Smoking status & & & \\
$\quad$ Yes & $85(45.70)$ & $92(48.94)$ & 0.531 \\
$\quad$ No & $101(54.30)$ & $96(51.06)$ & \\
\hline
\end{tabular}

Significant values are written in italics.

5'-CTGACTATCAATCACATCGGAATG-3' (reverse). A primer pair of rs17524488 was $5^{\prime}$-TGTCACTAGTGCCATTTGT-3' (forward) and $5^{\prime}$-TGTACCTTGGTCGGCGTTTG-3' (reverse). PCR was performed using $50 \mathrm{ng}$ DNA as a template under the following conditions: $95^{\circ} \mathrm{C}$ for $3 \mathrm{~min}$, then 35 cycles of $94^{\circ} \mathrm{C}$ for $30 \mathrm{~s}$, an annealing temperature at $60^{\circ} \mathrm{C}$ for $45 \mathrm{~s}$, and $72^{\circ} \mathrm{C}$ for $30 \mathrm{~s}$, with a final extension at $72^{\circ} \mathrm{C}$ for $10 \mathrm{~min}$. The PCR products were direct sequencing using an automated ABI 3100 DNA sequencer by GeneCore BioTechnologies (Shanghai, China).

2.4. OPN ELISA Assay. Serum concentration of OPN was measured by ELISA according to the protocol provided by the manufacturer (Raybiotech, Norcross, USA) in all participants. Serum was diluted as 1:10 into sample diluents. The optical density was measured at $450 \mathrm{~nm}$ (BioRad, USA).

2.5. Statistical Analysis. Statistical analysis was performed using SPSS 15.0 software. One way ANOVA and $t$-test were used to compare mean differences for continuous variables. Allele frequency was determined via direct counting. Binary logistic regression analysis was used to assess independent predictors of AS. Receiver operating characteristic curve (ROC) analysis was used to find the cut-off point of OPN for predicting AS. Differences in the distribution of genotypes between AS patients and control subjects were examined using the $\chi^{2}$ test, and a statistically significant difference was defined as $P<0.05$.

\section{Results}

3.1. Subject Characteristics. As was shown in Table 1, a total of 374 subjects were enrolled in this study, containing 186 cases and 188 controls. The clinical characteristics such as age, sex, body mass index (BMI), and smoking status had no significant difference between the case and control groups 
TABLE 2: Difference in the scores of BASDAI, BASFI, and BAS-G among AS patients stratified by different AS genotype.

\begin{tabular}{lccccc}
\hline SNP & Genotype & Number $(\%)$ & BASDAI & BASFI & BAS-G \\
\hline \multirow{3}{*}{9175 (exon 7) } & TT & $142(76.34)$ & $4.21 \pm 0.07$ & $2.79 \pm 0.07$ & $4.34 \pm 0.10$ \\
& TA & $44(23.66)$ & $4.30 \pm 0.12$ & $2.80 \pm 0.12$ & 0.965 \\
& Unadjusted $P$ value & & 0.561 & 0.18 \\
& Adjusted $P$ value & & 0.614 & 0.495 & 0.334 \\
rs17524488 & GG/GG & $102(54.84)$ & $4.20 \pm 0.08$ & $2.79 \pm 0.08$ & $4.37 \pm 0.12$ \\
& G/G & $84(45.16)$ & $4.27 \pm 0.09$ & $0.948 \pm 0.11$ & 0.961 \\
& Unadjusted $P$ value & & 0.529 & 0.988 & 0.821 \\
\hline
\end{tabular}

Data represent means \pm SEM, adjusted for the effects of age, sex, BMI, family history, smoking status, and disease duration.

TABLE 3: Risk factors for AS by binary logistic regression analysis.

\begin{tabular}{lccccc}
\hline & OR & $95 \%$ CL for OR & $P$ & OR $^{*}$ & $95 \%$ CL for OR $^{*}$ \\
\hline Sex & 1.065 & $0.710-1.599$ & 0.760 & $/$ & $/$ \\
Age & 0.981 & $0.959-1.003$ & 0.085 & $/$ & $/$ \\
BMI & 0.959 & $0.904-1.018$ & 0.167 & $/$ & $/$ \\
Family history & 2.277 & $1.108-4.682$ & 0.025 & $/$ & $/$ \\
Smoking status & 0.878 & $0.585-1.318$ & 0.531 & $/$ & $/$ \\
OPN & 1.011 & $1.007-1.014$ & 0.000 & 1.011 & $/$ \\
\hline
\end{tabular}

CI, confidence interval. Logistic regression models were used to calculate OR. * Adjusted for gender, age, BMI, family history, and smoking status. Significant values are written in italics.

(all $P>0.05$ ). Family history has significant difference between the case and control groups $(P<0.05)$. In AS subjects, all patients were HLA-B27 positive and their mean disease duration, mean BASDAI, mean BASFI, and mean BAS-G scores were $5.67 \pm 0.28,4.23 \pm 0.06,2.79 \pm 0.06$, and $4.38 \pm 0.09$, respectively.

3.2. Association of OPN Genetic Polymorphisms with the Disease Activity Index in AS Patients. We analyzed the relationship between disease activity index (BASDAI, BASFI, and BAS-G) and the two polymorphisms of OPN in AS patients. No significant association between $O P N$ polymorphisms and BASDAI, BASFI, or BAS-G (all $P>0.05$ ) was found. However, we failed to improve the significance even after adjustment for the effects of age, sex, BMI, smoking status, and family history (Table 2).

3.3. Increased Levels of OPN in AS Patients and Diagnostic Value of OPN for AS. Serum concentration of OPN was measured in 186 AS patients and 188 ethnically matched healthy controls by ELISA, even after adjusting for confounding risk factors (age, sex, BMI, smoking status, and family history), which shows that AS patients displayed significantly higher OPN serum levels (median, 217.76; range, $102.02-468.65 \mathrm{ng} / \mathrm{mL}$ ) than the controls (median, 156.99; range, 51.56-321.54 ng/mL) in Figure 1 and Table $3(P<001)$.

Taking AS as the dependent variable, the risk factors (gender, age, BMI, family history, and smoking status) were entered into binary logistic regression analysis. After adjusting for the risk factors, plasma levels of OPN remained with a significant association with an increased odds ratio (OR) for AS $(P<0.001)$. ROC curve analysis was performed to

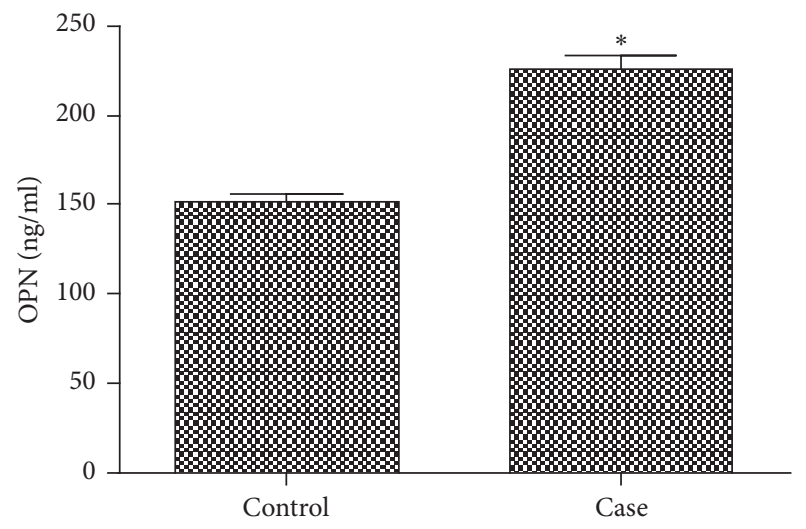

FIGURE 1: Serum concentration of OPN in AS patients and healthy controls. Compared with healthy controls, the serum levels of OPN in AS patients were significantly elevated $\left({ }^{*} P<0.01\right)$.

verify the diagnostic accuracy of OPN for AS. The area under curve (AUC) of OPN was 0.71 (95\% confidence intervals (CI) $0.662-0.764, P<0.001)$ and the optimal cut-off point for siglec-5 was $131.7 \mathrm{ng} / \mathrm{mL}$. At this level, the Youden index = 0.313 , sensitivity was $42.2 \%$ (95\% CI $0.349-0.494)$; specificity was $89.25 \%$ (95\% CI $0.839-0.933)$. The AUC of OPN + confounding risk factors was 0.73 (95\% CI $0.676-0.776, P<$ $0.001)$, which was higher than that of OPN, but this did not reach the level of statistical significance $(P>0.05)$ (Figure 2).

3.4. OPN Polymorphisms Are Associated with AS Patients. DNA fragments from 9041 to 9292 in exon 7 of the OPN gene and one known polymorphism (rs17524488) were analyzed 
TABLE 4: Frequencies of genotypes and alleles of OPN in AS patients and healthy control.

\begin{tabular}{|c|c|c|c|c|}
\hline Genotype/allele & Case $(n=186), n(\%)$ & Control $(n=188), n(\%)$ & OR $(95 \% \mathrm{CL})$ & $P$ \\
\hline \multicolumn{5}{|l|}{9175 (exon 7) } \\
\hline \multicolumn{5}{|l|}{ Genotype } \\
\hline TT & $142(76.34)$ & $164(87.23)$ & \multirow{2}{*}{$1.853(1.177-2.918)$} & \multirow{2}{*}{0.006} \\
\hline $\mathrm{TA}$ & $44(23.66)$ & $24(12.77)$ & & \\
\hline \multicolumn{5}{|l|}{ Allele } \\
\hline $\mathrm{T}$ & $328(88.17)$ & $352(93.62)$ & \multirow{2}{*}{$1.853(1.151-2.983)$} & \multirow{2}{*}{0.01} \\
\hline $\mathrm{A}$ & $44(11.83)$ & $24(6.38)$ & & \\
\hline \multicolumn{5}{|l|}{ rs17524488 } \\
\hline \multicolumn{5}{|l|}{ Genotype } \\
\hline GG/GG & $102(54.84)$ & $124(65.96)$ & \multirow{2}{*}{$1.327(1.029-1.711)$} & \multirow{2}{*}{0.028} \\
\hline $\mathrm{G} / \mathrm{G}$ & $84(45.16)$ & $64(34.04)$ & & \\
\hline \multicolumn{5}{|l|}{ Allele } \\
\hline GG & $204(54.84)$ & $248(65.96)$ & \multirow{2}{*}{$1.327(1.108-1.588)$} & \multirow{2}{*}{0.002} \\
\hline G & $168(45.16)$ & $128(34.04)$ & & \\
\hline
\end{tabular}

Significant values are written in italics.

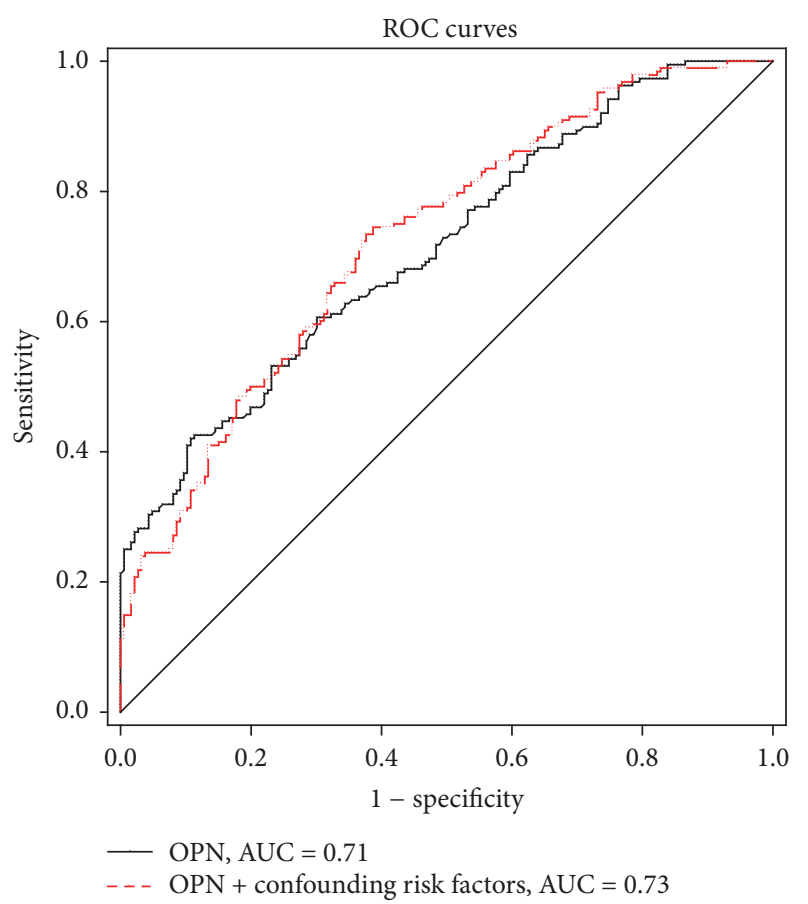

FIgure 2: Analysis of ROC curve to detect OPN in AS patients. In ROC analysis, the AUC of Siglec-5 was 0.71; the AUC of confounding risk factors was 0.73 .

using direct sequencing in 186 AS patients and 188 ethnically matched healthy controls. We found a heterozygous, novel $9175 \mathrm{~T}>\mathrm{A}$ in exon 7 of the OPN gene, and there is a small insertion at nt-156 (rs17524488), which has only two alleles: G/G and GG/GG. Overall distributions of genotypes of OPN gene 9175 and rs17524488 were significantly different in AS patients controls (all $P<0.005$ ). Frequencies of allele A and allele $G$ were all higher in AS patients than in the controls (all $P<0.005$ ), indicating that subjects who carried allele A or allele $G$ have a significantly higher risk of developing

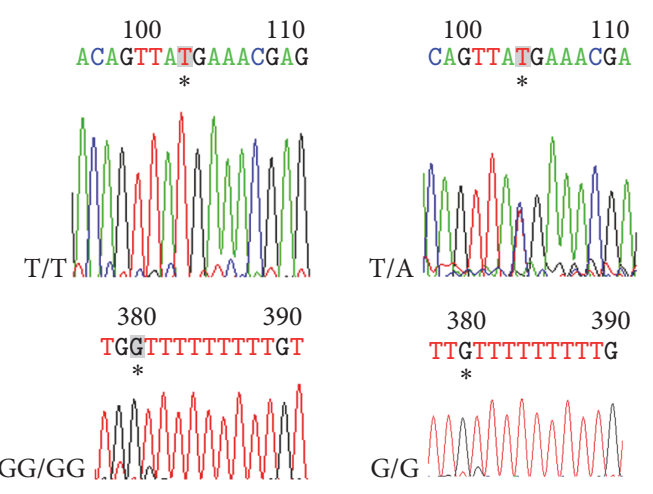

FIGURE 3: The results of DNA sequencing.

AS (Table 4). The results of DNA sequencing were shown in Figure 3.

3.5. OPN Polymorphisms Are Associated with the OPN Levels. As shown in Figure 4, subjects carrying the rs17524488 G/G genotype of the OPN display significantly higher OPN serum levels than the GG/GG genotype $\left({ }^{*} P<0.05\right)$, but there were no significant associations between TT or TA genotypes with OPN serum levels in healthy controls. However, there were no significant associations between OPN genotypes and OPN serum levels in AS patients.

\section{Discussion}

In this study, a heterozygous, novel $9175 \mathrm{~T}>\mathrm{A}$ in exon 7 of the $O P N$ gene and rs17524488 were significantly associated with a genetic predisposition for AS. Patients with AS had higher serum levels of OPN compared with controls, even after adjusting for confounding risk factors. However, the rs17524488 G/G genotype of the OPN was significantly correlated with an increased OPN serum level compared to 


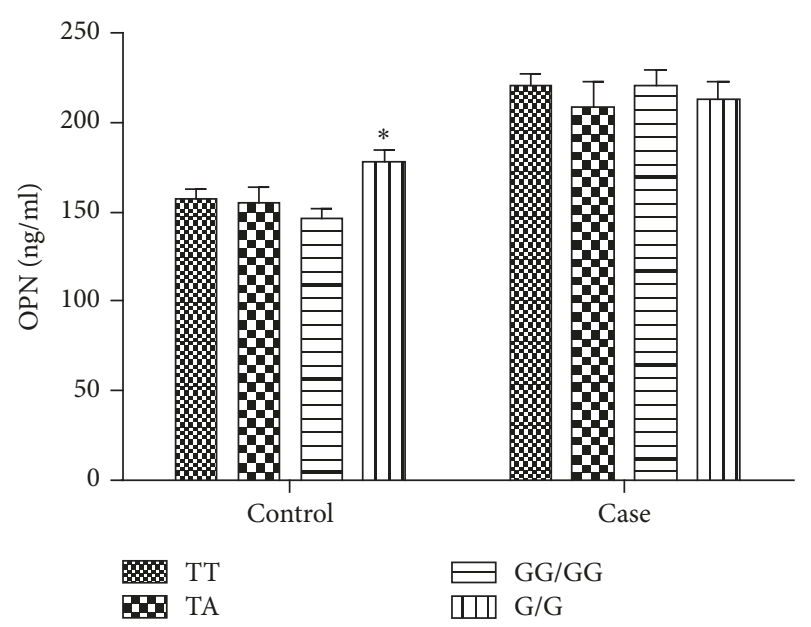

FIGURE 4: OPN polymorphisms are associated with the OPN levels. In healthy controls, subjects carrying the rs17524488 G/G genotype of the OPN display significantly higher OPN serum levels than the GG/GG genotype $\left({ }^{*} P<0.05\right)$.

the GG/GG genotype in healthy controls, which suggests that $\mathrm{rs} 17524488 \mathrm{G} / \mathrm{G}$ genotype may be involved in the pathogenesis of AS. This is the first study to demonstrate a strong relationship between the 9175th- (exon 7) position polymorphism of $O P N$ or rs17524488 and AS patients.

We first considered the possibility that $O P N$ polymorphisms are related to the inflammatory process in AS. It has been reported that patients with AS had significantly higher plasma OPN, TNF-alpha, and IL-6 levels and more mRNA expression than healthy controls. The plasma OPN level was correlated with serum ALP, OCN, and CTX-I levels, but not with disease activity in AS. OPN might be involved in bone remodeling rather than in inflammation in AS [18]. In this study, no significant association between $O P N$ polymorphisms and disease activity index (all $P>$ 0.05) was found, and we also found that patients with AS had significantly higher plasma OPN than healthy controls which is consistent with them [18]. We found a heterozygous, novel $9175 \mathrm{~T}>\mathrm{A}$ in exon 7 of the OPN gene, and distributions of genotypes of OPN gene 9175 and rs17524488 were significantly different in AS patients controls. Frequencies of allele A and allele $\mathrm{G}$ were all higher in AS patients than in the controls, indicating that subjects who carried allele A or allele $\mathrm{G}$ have a significantly higher risk of developing AS (OR $=1.853$ or $\mathrm{OR}=1.327)$. In addition, subjects carrying the rs17524488 G/G genotype of the OPN display significantly higher OPN serum levels than the GG/GG genotype in healthy controls, strongly indicating that the rs $17524488 \mathrm{G} / \mathrm{G}$ genotype of the OPN may be involved in the pathogenesis of AS. Thus, the mechanism by which this polymorphism contributes to AS susceptibility is less clear. There is evidence suggesting that OPN acts as a proinflammatory cytokine and plays an important role in regulating inflammation [18]; subjects carrying polymorphism loci of the $O P N$ will be more sensitive to the immune response; in addition, it might be relevant in the regulation of OPN production in response to the initial immunostimulating trigger.
So far, genetic variants in the $O P N$ gene have shown being involved in susceptibility to other immune-mediated diseases such as systemic lupus erythematosus [19], Crohn's disease [20], rheumatoid arthritis [21], and lupus nephritis [22]. In addition, overexpression of OPN has been described in several basic inflammatory processes, such as arthritis [23], myocardial remodeling after infarction [24], kidney interstitial fibrosis after obstructive uropathy [25], wound healing [26], and several types of cancer [27], where it is demonstrated that OPN gene polymorphism and OPN levels play important roles in immune- and inflammatorymediated diseases, including AS.

There are some limitations of the present study that should be considered. Large sample study needs to be explored further. Although this case-control study revealed a strong relationship between the 9175th- (exon 7) position polymorphism of OPN or rs17524488 and AS patients, the precise molecular mechanism underlying the influence of OPN polymorphisms on the development of AS remains to be determined in further prospective studies.

\section{Conclusions}

In conclusion, plasma OPN level is implicated as an early diagnostic marker of AS; the novel 9175th- (exon 7) position polymorphism of OPN or rs17524488 was related to susceptibility to AS in a Chinese population; the rs17524488 G/G genotype may be involved in the pathogenesis of AS, and the precise molecular mechanism underlying the influence of OPN polymorphisms on the development of AS remains to be determined in further prospective studies.

\section{Conflicts of Interest}

No conflicts of interest are associated with this work.

\section{Authors' Contributions}

Aiping Deng and Guoliang Yang conceived and designed the experiments. Juyi Li, Yi Cai, and Zhongjing Wang performed the experiments. Juyi Li, Zhongjing Wang, and Guoliang Yang analyzed the data. Juyi Li and Aiping Deng wrote the paper. Juyi Li and Yi Cai contributed equally to this paper.

\section{Acknowledgments}

The authors thank all the volunteers that participated in this study. This study was supported by Grants from the Health and Family Planning Commission of Wuhan Municipality (WX17B02) and Grants from the Health and Family Planning Commission of Hubei Province (WJ2017M183).

\section{References}

[1] P. Castro-Santos, M. A. Gutiérrez, and R. Díaz-Peña, "Genética, HLA-B27 y espondilitis anquilosante: 40 años," Revista Médica de Chile, vol. 142, no. 9, pp. 1165-1173, 2014.

[2] B. Xu, J.-X. Ma, X.-L. Ma, H.-B. Jia, R. Feng, and L.-Y. Xu, "Association between rs7517847 and rs2201841 polymorphisms 
in IL-23 receptor gene and risk of ankylosing spondylitis: A meta-analysis," PeerJ, vol. 2015, no. 3, article no. e910, 2015.

[3] N. Haroon and R. D. Inman, "Ankylosing spondylitis - new criteria, new treatments," Bulletin of the NYU Hospital for Joint Diseases, vol. 68, pp. 171-174, 2010.

[4] Q. Zeng, R. Chen, J. Darmawan et al., "Rheumatic diseases in China," Arthritis Research \& Therapy, vol. 10, no. 1, p. R17, 2008.

[5] N. G. Wang, F. Wang, B. Y. Tan, S. J. Han, J. Dong, Z. N. Yuan et al., "Genetic analysis of Tnfst15 variants in ankylosing spondylitis," International Journal of Clinical and Experimental Pathology, vol. 8, pp. 15210-15215, 2015.

[6] R. Max, H. M. Lorenz, and F. Mackensen, "Ocular involvment in spondyloarthropathies: HLA B27 associated uveitis," Zeitschrift für Rheumatologie, vol. 69, no. 5, pp. 397-402, 2010.

[7] Y. Ou, "Anthrax toxin receptor 2 gene (ANTXR2) rs4333130 is associated with ankylosing spondylitis," International Journal of Clinical and Experimental Medicine, vol. 8, no. 5, pp. 7679-7683, 2015.

[8] W. Chai, Z. Lian, C. Chen, J. Liu, L. L. Shi, and Y. Wang, "JARID1A, JMY, and PTGER4 Polymorphisms Are Related to Ankylosing Spondylitis in Chinese Han Patients: A CaseControl Study," PLoS ONE, vol. 8, no. 9, Article ID e74794, 2013.

[9] K. X. Wang and D. T. Denhardt, "Osteopontin: role in immune regulation and stress responses," Cytokine \& Growth Factor Reviews, vol. 19, no. 5-6, pp. 333-345, 2008.

[10] G. Murugaiyan, A. Mittal, and H. L. Weiner, "Increased osteopontin expression in dendritic cells amplifies IL-17 production by CD4+ T cells in experimental autoimmune encephalomyelitis and in multiple sclerosis," The Journal of Immunology, vol. 181, no. 11, pp. 7480-7488, 2008.

[11] F. Giacopelli, R. Marciano, A. Pistorio et al., "Polymorphisms in the osteopontin promoter affect its transcriptional activity," Physiological Genomics, vol. 20, pp. 87-96, 2005.

[12] C.-C. Liu, S.-P. Huang, L.-Y. Tsai et al., "The impact of osteopontin promoter polymorphisms on the risk of calcium urolithiasis," Clinica Chimica Acta, vol. 411, no. 9-10, pp. 739743, 2010.

[13] D. Hendig, M. Arndt, C. Szliska, K. Kleesiek, and C. Götting, "SPPI promoter polymorphisms: Identification of the first modifier gene for pseudoxanthoma elasticum," Clinical Chemistry, vol. 53, no. 5, pp. 829-836, 2007.

[14] C. Lv, Y. Li, J. Xu et al., "Association of SPP1 promoter variants with hip osteoarthritis susceptibility in Chinese population," Gene, vol. 564, no. 1, pp. 9-13, 2015.

[15] J. Liu, C. He, Q. Yuan, Z. Wang, C. Xing, and Y. Yuan, "Association between promoter polymorphisms of OPN gene and cancer risk: A meta-analysis," OncoTargets and Therapy, vol. 9, pp. 87-97, 2015.

[16] B. Cheema, S. Iyengar, R. Sharma, H. Kohli, A. Bhansali, and M. Khullar, "Association between Osteopontin Promoter Gene Polymorphisms and Haplotypes with Risk of Diabetic Nephropathy," Journal of Clinical Medicine, vol. 4, no. 6, pp. 1281-1292, 2015.

[17] J. C.-C. Wei, R.-H. Wong, J.-H. Huang et al., "Evaluation of internal consistency and re-test reliability of Bath ankylosing spondylitis indices in a large cohort of adult and juvenile spondylitis patients in Taiwan," Clinical Rheumatology, vol. 26, no. 10, pp. 1685-1691, 2007.

[18] S. T. Choi, J. H. Kim, E.-J. Kang et al., "Osteopontin might be involved in bone remodelling rather than in inflammation in ankylosing spondylitis," Rheumatology, vol. 47, no. 12, pp. 17751779, 2008.
[19] A. P. Xu, J. Bai, J. Lu, Y. Y. Liang, J. G. Li, D. Y. Lai et al., "Osteopontin gene polymorphism in association with systemic lupus erythematosus in Chinese patients," Chinese Medical Journal, vol. 120, pp. 2124-2128, 2007.

[20] J. Glas, J. Seiderer, C. Bayrle et al., "The role of osteopontin (OPN/SPP1) haplotypes in the susceptibility to Crohn's disease," PLoS ONE, vol. 6, no. 12, Article ID e29309, 2011.

[21] E. Urcelay, A. Martínez, A. Mas-Fontao et al., "Osteopontin gene polymorphisms in spanish patients with rheumatoid arthritis," The Journal of Rheumatology, vol. 32, no. 3, pp. 405-409, 2005.

[22] S. Salimi, M. Noora, S. Nabizadeh et al., "Association of the osteopontin rs1126616 polymorphism and a higher serum osteopontin level with lupus nephritis," Biomedical Reports, vol. 4, no. 3, pp. 355-360, 2016.

[23] N. Yamamoto, F. Sakai, S. Kon et al., "Essential role of the cryptic epitope SLAYGLR within osteopontin in a murine model of rheumatoid arthritis," The Journal of Clinical Investigation, vol. 112, no. 2, pp. 181-188, 2003.

[24] A. Tamura, M. Shingai, N. Aso, T. Hazuku, and M. Nasu, "Osteopontin is released from the heart into the coronary circulation in patients with a previous anterior wall myocardial infarction," Circulation Journal, vol. 67, no. 9, pp. 742-744, 2003.

[25] Y. Xie, M. Sakatsume, S. Nishi, I. Narita, M. Arakawa, and F. Gejyo, "Expression, roles, receptors, and regulation of osteopontin in the kidney," Kidney International, vol. 60, no. 5, pp. 1645-1657, 2001.

[26] D. T. Denhardt and S. R. Rittling, "Osteopontin function in pathology: Lessons from osteopontin-deficient mice," Experimental Nephrology, vol. 7, no. 2, pp. 103-113, 1999.

[27] Y. Mi, K. Ren, F. Dai, L. Zhu, and N. Feng, "OPN gene polymorphisms, rs17524488 GG/G, rs11730582 T/C, and rs9138 $\mathrm{C} / \mathrm{A}$, and cancer risk in a Chinese population," Scientific Reports, vol. 5, Article ID 14164, 2015. 


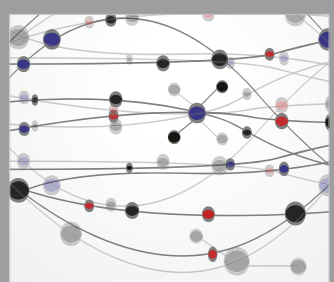

The Scientific World Journal
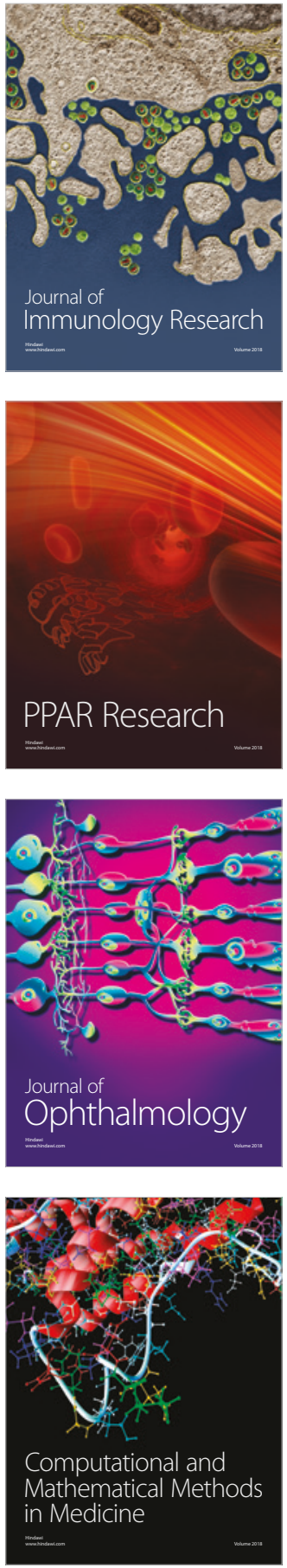

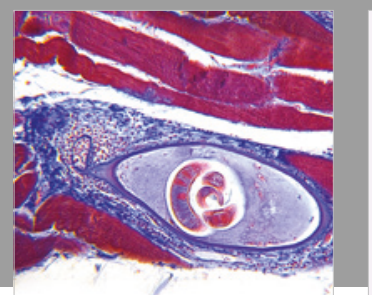

Gastroenterology Research and Practice

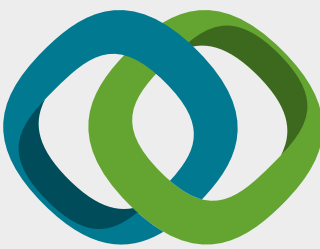

\section{Hindawi}

Submit your manuscripts at

www.hindawi.com
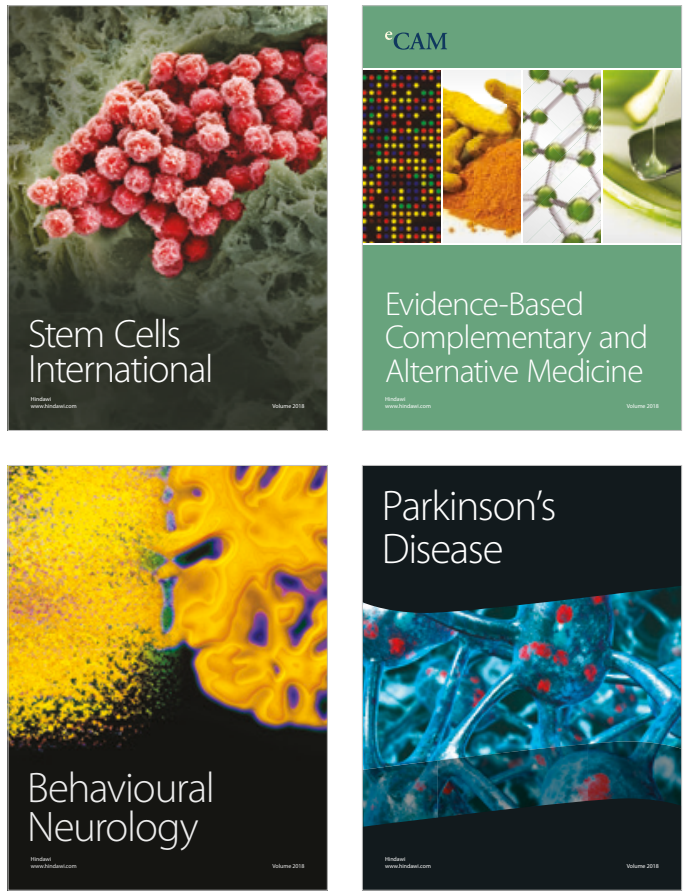

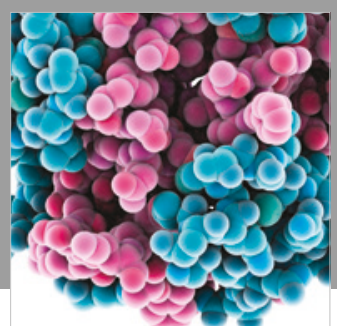

ournal of

Diabetes Research

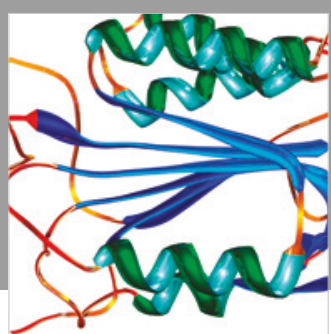

Disease Markers
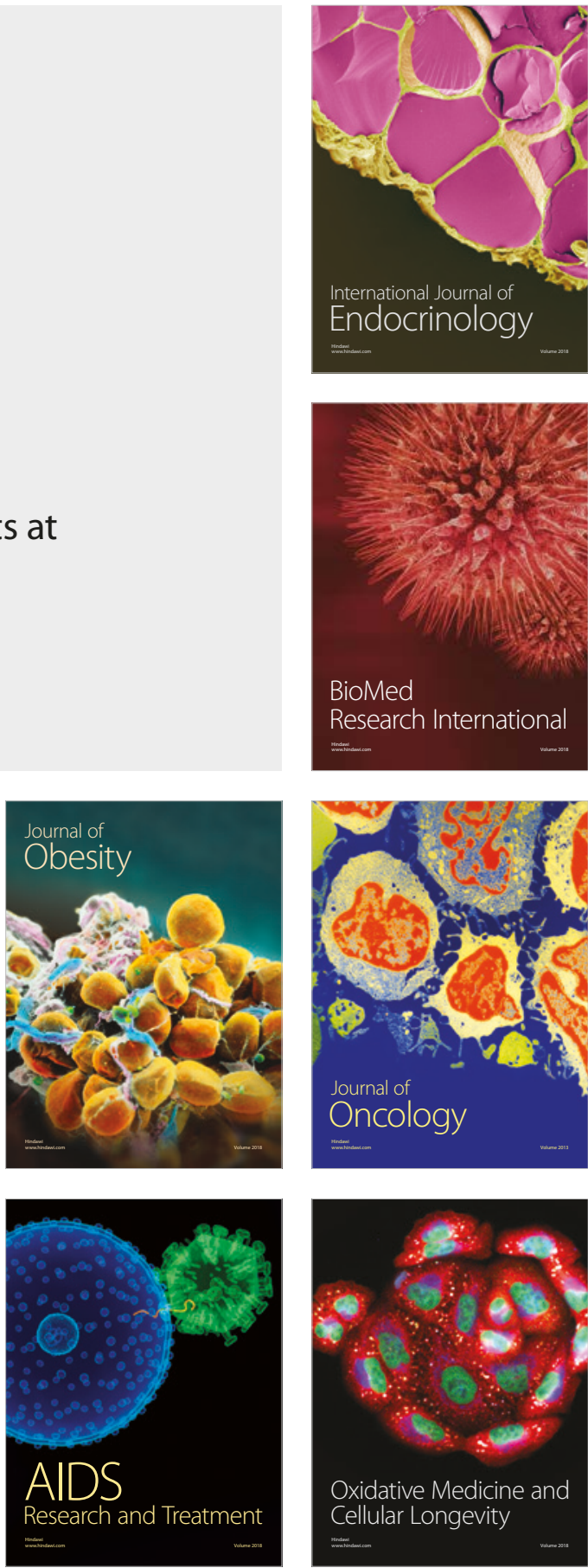